\title{
Spatial Akhmediev breathers and modulation instability growth-decay cycles in a quadratic optical medium
}

\author{
Roland Schiek $\oplus^{1, *}$ and Fabio Baronio ${ }^{2, \dagger}$ \\ ${ }^{1}$ Ostbayerische Technische Hochschule Regensburg, Prüfeninger Strasse 58, 93049 Regensburg, Germany \\ ${ }^{2}$ Istituto Nazionale di Ottica CNR and Dipartimento di Ingegneria dell'Informazione, Università di Brescia, \\ Via Branze 38, 25123 Brescia, Italy
}

(Received 11 May 2019; revised manuscript received 28 August 2019; published 12 December 2019)

\begin{abstract}
We investigate the spatial nonlinear localization of light on a quasi-plane-wave background with a harmonic perturbation induced by modulation instability in a quadratic nonlinear optical medium. In particular, we demonstrate experimentally the excitation of deterministic Akhmediev breathers and thus the growth-decay dynamics of modulation instability in a $\mathrm{LiNbO}_{3}$ slab waveguide. The results should stimulate new interest in modulation instability, extreme events, turbulence, recurrence, and supercontinuum generation in quadratic nonlinear optics.
\end{abstract}

DOI: 10.1103/PhysRevResearch.1.032036

Introduction. Instabilities and chaotic dynamics occur frequently in a wide class of wave phenomena when intense waves propagate through a nonlinear medium [1]. One salient form of instability in a distributed nonlinear system is its response to a harmonic modulation. Such instability is generally known as modulation instability (MI) [2,3]. MI leads to the exponential growth of an initial harmonic perturbation at the expense of a strong pump background. MI dynamics has been deeply investigated since the early days of nonlinear optics [4] but it has remained a somewhat complex and mysterious process. The breakup and self-filamentation of an optical beam as a result of MI was one of the first nonlinear spatial effects observed in the 1960s and early 1970s [5]. The temporal breakup of a continuous wave (cw) field as a result of MI was observed in an optical fiber in the 1980s [6]. Dynamical MI was demonstrated in photorefractive and quadratic nonlinear crystals in the 1990s $[7,8]$.

Nowadays, the investigation of MI in nonlinear optics is once again the subject of tremendous interest boosted by the strong link of MI with the formation of deterministic breathers $[9,10]$, statistics of anomalous waves [11,12], turbulence $[13,14]$, supercontinuum generation, and frequency combs [15]. Of great importance has been the discovery that the dynamics of MI can be described by analytic families of periodic solutions, in particular Akhmediev breather solutions in cubic nonlinear media [16]. In fact, Akhmediev breathers describe the MI growth-decay cycle of unstable waves starting from a regular background, significantly enhancing the wave

\footnotetext{
*roland.schiek@oth-regensburg.de

†fabio.baronio@unibs.it
}

Published by the American Physical Society under the terms of the Creative Commons Attribution 4.0 International license. Further distribution of this work must maintain attribution to the author(s) and the published article's title, journal citation, and DOI. amplitudes during the envelope compression until reaching a precise saturation point, and finally declining the envelope back to a regular state. Moreover, the growth-decay dynamics in breathers constitutes a manifestation of the universal nonlinear phenomenon known as Fermi-Pasta-Ulam (FPU) recurrence. Deterministic Akhmediev breathers, MI growthdecay cycles, and FPU recurrence have been experimentally characterized and demonstrated in the temporal domain in cubic nonlinear fiber optics with long interaction lengths [9,10,12-15,17-20]. Very recently, FPU recurrence and its exact dynamics have also been demonstrated in the spatial domain in cubic nonlinear photorefractive crystals [21].

However, observations of breather growth-decay dynamics in the spatial domain based on fast electronic nonlinearities have been elusive so far because the small cubic nonlinearities in common crystals would require enormous intensities. In noncentrosymmetric crystals the intensity requirements can be reduced by a proper enhancement of the effective nonlinear refraction due to cascaded quadratic nonlinearities [22]. The existence of Akhmediev breathers and Peregrine walking solitons has indeed been recently predicted in the regime of quadratic cascading $[23,24]$. This system is especially interesting because just by tuning the wave-vector mismatch of the quadratic nonlinear interaction we can adjust the degree of approximation in describing the quadratic nonlinear system with a cubic nonlinear model providing experimental information on the robustness of breather solutions against system disturbances.

In this Rapid Communication, we investigate the spatial nonlinear localization of light on a quasi-plane-wave background with a harmonic modulation in a $\mathrm{LiNbO}_{3}$ slab waveguide. We experimentally unveil the existence of deterministic Akhmediev spatial breather-like beams and thus the MI growth-decay cycle in a quadratic waveguide system where the waveguide mode is coupled to its second harmonic. On the one hand, our results pave the way to the reinterpretation of quadratic MI dynamics in terms of shaping-invariant 
solitons and breathers instead of only solitons as essential actors of MI phenomena [8,25-28]. On the other hand, our system constitutes a renewed spatial platform for the investigation of light localization, offering with simple camera observation new possibilities to investigate extreme MI events and the recurrence and robustness of breather solutions.

Experimental and theoretical methods. We considered spatial light dynamics in a titanium indiffused $\mathrm{LiNbO}_{3} \mathrm{Y}$-cut slab waveguide with a length $L=47 \mathrm{~mm}$ which was fabricated in the 1990s by Sohler's group in Paderborn for the experimental demonstration of quadratic solitons [27] and MI measurements [28]. Type I second-harmonic generation (SHG) can be implemented by coupling a fundamental frequency $(\mathrm{FF})$ ordinary polarized transverse magnetic $\left(\mathrm{TM}_{0}\right)$ mode at a wavelength of $1.32 \mu \mathrm{m}$ to extraordinary transverse electric (TE) modes at the second-harmonic ( $\mathrm{SH}$ ) frequency at a wavelength of $0.66 \mu \mathrm{m}$. Temperature tuned birefringent phase matching occurs at $297^{\circ} \mathrm{C}$ to the $\mathrm{TE}_{0}$ mode and at $344{ }^{\circ} \mathrm{C}$ to the $\mathrm{TE}_{1}$ mode. For controlling the phase matching, the crystal was housed in an oven. The waves propagate along the $z$ direction and are guided along the $y$ coordinate, and the light localization is observed in the $x$ direction.

Theoretical modeling is based on the standard description of SHG with one resonance in a slab waveguide [27]. The electric field beams $E_{1,2}(x, y, z)$ at the $\mathrm{FF}$ and $\mathrm{SH}$ frequency $\omega_{1,2}\left(\omega_{2}=2 \omega_{1}\right)$ are expressed as $E_{1,2}(x, y, z)=$ $e_{1,2}(y) a_{1,2}(x, z)$ where $e_{1,2}$ are the waveguide modes normalized to a unit power flow per film width $\left(p_{0}=1 \mathrm{~W} / \mathrm{m}\right)$; $a_{1,2}$ are the effective beam envelopes with $I_{1,2}=\left|a_{1,2}\right|^{2} p_{0}$ representing the power flow per film width. The beams $a_{1,2}$ obey the nonlinear, not integrable, coupled equations:

$$
\begin{aligned}
\frac{\partial a_{1}}{\partial z}+i \frac{1}{2 k_{1}} \frac{\partial^{2} a_{1}}{\partial x^{2}}= & -i \frac{\omega_{1} K^{(2)} 2 d}{4 p_{0}} a_{1}^{*} a_{2} e^{i \Delta k z} \\
& -i \frac{\omega_{1} K^{(3)} \chi^{(3)}}{4 p_{0}}\left|a_{1}\right|^{2} a_{1}-\frac{\alpha_{1}}{2} a_{1}, \\
\frac{\partial a_{2}}{\partial z}+i \frac{1}{2 k_{2}} \frac{\partial^{2} a_{2}}{\partial x^{2}}= & -i \frac{2 \omega_{1} K^{(2)} d}{4 p_{0}} a_{1} a_{1} e^{-i \Delta k z}-\frac{\alpha_{2}}{2} a_{2} .
\end{aligned}
$$

$k_{1,2}$ are the wave numbers, $\Delta k=2 k_{1}-k_{2}$ is the wave-vector mismatch. The mode overlap integrals $K^{(2)}=\int e_{1} e_{1} e_{2}^{*} d y$ and $K^{(3)}=\int e_{1} e_{1} e_{1}^{*} e_{1}^{*} d y$ determine the nonlinear strengths together with the nonlinear susceptibilities $d$ and $\chi^{(3)} \cdot \alpha_{1}$ and $\alpha_{2}$ describe losses. All parameters have been measured [29] (see Refs. [27,28,30] for waveguide details). The intrinsic cubic nonlinearity for the FF wave has also been considered in Eqs. (1) because of the large experimental peak intensities. We emphasize that in reality, $\mathrm{SH}$ resonances to both the $\mathrm{SH}$ $\mathrm{TE}_{0}$ and $\mathrm{TE}_{1}$ modes contribute to the effective nonlinear refraction. However, the two resonances are well separated. Consequently, the dominant one with the smaller phase mismatch can be described with the SH Eqs. (1) while the other is considered only approximately by adding its effective cubic nonlinearity to the $\chi^{(3)}$ term.

Far from the phase-matching conditions, neglecting losses, Eqs. (1) may be reduced to a nonlinear Schrödinger (NLS) equation for the FF beam [27]:

$$
\frac{\partial a_{1}}{\partial z}+i \frac{1}{2 k_{1}} \frac{\partial^{2} a_{1}}{\partial x^{2}}=-i \gamma\left|a_{1}\right|^{2} a_{1}
$$

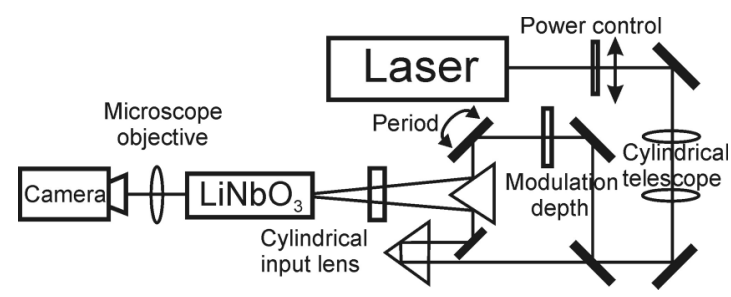

FIG. 1. Experimental setup.

where the effective cubic nonlinearity reads $\gamma=$ $\frac{1}{\Delta k}\left(\frac{\omega_{1} K^{(2)} 2 d}{4 p_{0}}\right)^{2}+\frac{\omega_{1} K^{(3)} \chi^{(3)}}{4 p_{0}}$. Varying the temperature, the wave-vector mismatch is controlled and thus the cascading contribution. Under the condition $\gamma>0$ Akhmediev breathers are solutions of Eq. (2). With the normalization $\psi=a_{1} / a_{1 p}, \quad s=x \sqrt{\gamma a_{1 p}^{2} k_{1}}$, and $\xi=z \gamma a_{1 p}^{2}$, where $a_{1 p}$ describes the plane-wave background intensity $a_{1 p}^{2} p_{0}=I_{1 \mathrm{P}}$, the well-known analytical form of the Akhmediev breather is obtained (see Ref. [23]):

$\psi$

$$
=e^{-i \xi}\left[\frac{(1-4 a) \cosh (b \xi)+\sqrt{2 a} \cos (\Omega s)-i b \sinh (b \xi)}{\cosh (b \xi)-\sqrt{2 a} \cos (\Omega s)}\right] .
$$

$\Omega$ is the normalized modulation frequency. $a=\frac{1}{2}\left(1-\Omega^{2} / 4\right)$ determines the frequencies that experience gain when $0<$ $a<\frac{1}{2} . b=\sqrt{8 a(1-2 a)}$ is the growth factor of the instability. Thus, exploiting Akhmediev breather solutions (3) and the transformation mapping to Eq. (2), the FF breather dynamics of Eqs. (1) should exist in the $x-z$ plane of the quadratic slab waveguide.

Breather excitation and measurement technique. Experimentally, breathers can be deterministically excited from an unstable quasiconstant background with an initial finiteamplitude periodic perturbation with amplitude and phase pattern equal to the breather field at a fixed $\xi$ before the breather maxima. We used a frequency-doubled Nd:YAG-pumped optical parametric amplifier (OPA) with cw-seeding delivering 5.5-ps-long pulses at a wavelength of $1.32 \mu \mathrm{m}$ with up to $450 \mathrm{~kW}$ peak power. With a cylindrical telescope the laser beam was transformed into a very wide Gaussian elliptical beam $(1.4 \mathrm{~mm} \times 8 \mu \mathrm{m})$ providing the background. The beam was end-fire coupled into the FF $\mathrm{TM}_{0}$ mode. Power scaling with calibrated power meters enabled absolute measurements of the average input power. The input power is converted to the temporal peak intensity $I_{1 \mathrm{P}}$ in the mode in the center of the input beam. A few $\%$ of the FF beam were separated after the telescope and coupled with a tilt and good overlap to the main FF beam into the waveguide. The interference between the two FF beams produced a spatial modulation of the input with adjustable period and modulation depth $m=\left(I_{1, \max }-I_{1, \min }\right) / I_{1, \max }$. Figure 1 shows a sketch of the experimental setup. The beam width of $1.4 \mathrm{~mm}$ along the $x$ coordinate can be considered large enough in the compromise between available power and infinite beam width so that a FF beam with a transverse modulation with periods between 130 and $300 \mu \mathrm{m}$ well approximates a quasiconstant background 
with a harmonic modulation which would approximate the amplitude pattern but not the phase profile of a straight propagating breather in its growing stage. This problem has previously been solved with the input of a second tilted beam [21]. However, as discussed in Ref. [31] our one-sideband excitation has an important advantage: The missing sideband is generated shortly after the input. Contrary to the twosideband excitation where the phase between the harmonic modulation and the background needs to be controlled, our one-sideband excitation is phase insensitive. Measured spatial spectra showed the generation of the missing sideband. As predicted, we did not observe a dependence on the phase of the tilted input beam.

Because the pure Akhmediev breather in Eq. (3) is a separatrix between two doubly periodic breather solution families $[19,32]$ it can never be observed in that purity. Inevitable small perturbations of the input force the beam evolution into these solutions with closed trajectories in the complex field plane near the heteroclinic orbits of the pure Akhmediev breather, reducing the ideally infinite $\xi$ periodicity to finite values in all breather experiments. In addition, it has been suggested that systems with growing deviations from an ideal NLS model have solutions with amplitude-phase trajectories deviating increasingly from the closed loops of the doubly periodic solutions $[32,33]$. However, the doubly periodic solutions are pretty robust and for our approximately NLS system with approximative breather initial conditions we find easily parameters where we observe at least one cycle of breather localization where the field trajectory is still close to the heteroclinic orbit of the Akhmediev breather. So we can characterize the main cycle of a breather where the light is highly localized between areas with growing and decaying MI.

Our sample is housed in an oven and we do not have access to the spatial dynamics of the beam along the evolution $z$ coordinate. We can only observe the beam at the input and output of the waveguide by imaging into cameras. Nevertheless, it has been demonstrated that the breather dynamics may be characterized by varying the input intensity [9] or nonlinearity [21]. For given modulation depth and intensity of the excitation the breather maxima develop at a specific and analytically predicted distance from the input $[31,34]$. For increasing intensity this distance decreases. Therefore, every scan of a breather at a desired $\xi$ can be sampled at the waveguide output facet just by changing the intensity as long as it exceeds the threshold for MI. Each scan is normalized with respect to its power. The composition of the normalized scans visualizes self-localization of light in breathers. Strictly, each scan for a new intensity belongs to a different breather with modified parameters $\Omega, a$, and $b$. A slight readjustment of the experimental spatial periodicity could keep these parameters constant yielding - from theory known - the breather pictures with constant normalized modulation frequency. However, experimental results are preferably presented for constant real-world modulation frequency, preventing the need of changing simultaneously two parameters in one measurement set.

Results and discussion. In the cascading regimes, we succeeded in exciting clean Akhmediev breather dynamics. Figures 2-4 report typical normalized spatial FF beam (a)

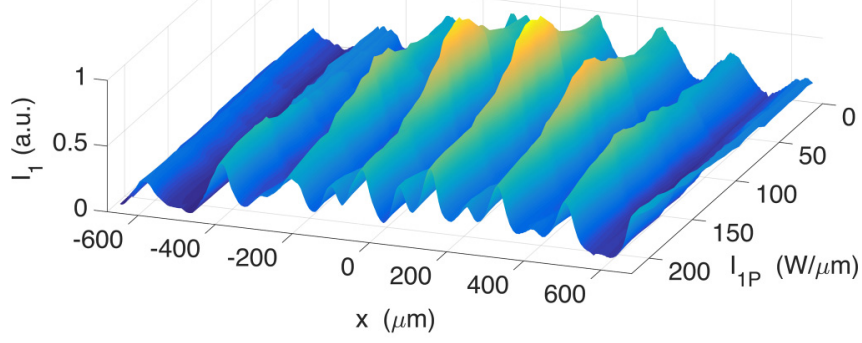

(b)

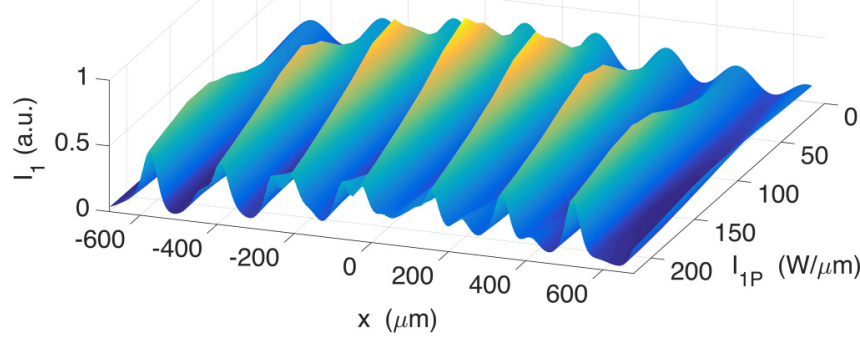

FIG. 2. Normalized intensity distribution $I_{1}\left(x, I_{1 \mathrm{P}}\right)$ of the FF beam at the waveguide output: (a) measurement and (b) simulation, $T=287.5^{\circ} \mathrm{C}, \Delta k L=113 \pi$ at the $\mathrm{TE}_{0}$ resonance, modulation pe$\operatorname{riod} 185 \mu \mathrm{m}, m=0.6$.

characterizations at the waveguide output along the $x$ coordinate varying the FF input intensity $I_{1 \mathrm{P}}$.

In Fig. 2 at a temperature of $T=287.5^{\circ} \mathrm{C}$ quadratic cascading contributes $63 \%$ to the total effective cubic nonlinearity $\gamma$, with $51 \%$ from the $\mathrm{TE}_{0}$ and $12 \%$ from the $\mathrm{TE}_{1}$ resonance. The remaining 37\% come from the intrinsic $\chi^{(3)}$. Figure 2(a) refers to measurements and Fig. 2(b) to simulations based on Eqs. (1). The simulations take all experimental details without any fit parameters into account: the onesideband excitation, and waveguide details like mode characteristics, losses, nonuniformities, and material data. Only the pulsed excitation is considered by simple time averaging, neglecting temporal dispersive effects. This approximation
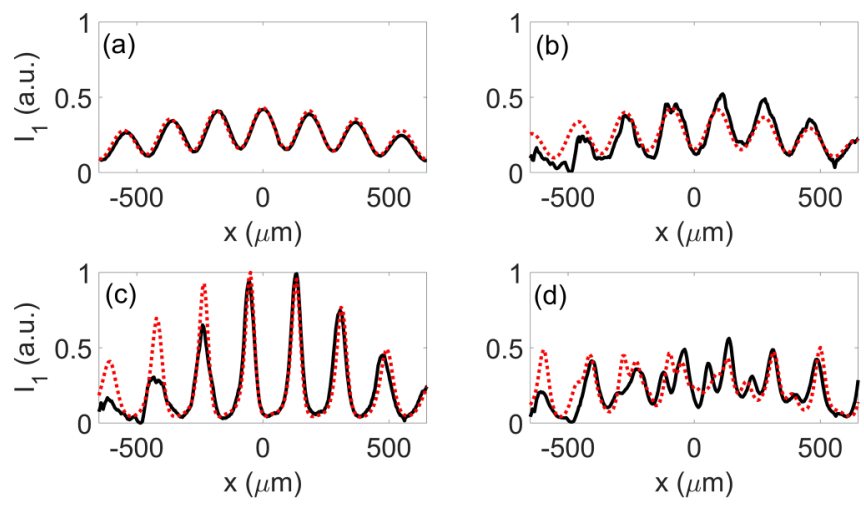

FIG. 3. Normalized intensity profiles $I_{1}(x)$ of the FF beam: (a) input spot, (b) waveguide output at $I_{1 \mathrm{P}}=7 \mathrm{~W} / \mu \mathrm{m}$, (c) $I_{1 \mathrm{P}}=$ $95 \mathrm{~W} / \mu \mathrm{m}$, and (d) $I_{1 \mathrm{P}}=215 \mathrm{~W} / \mu \mathrm{m}$ under the experimental conditions of Fig. 2. Black-solid lines indicate experimental and red-dotted lines numerical results. 

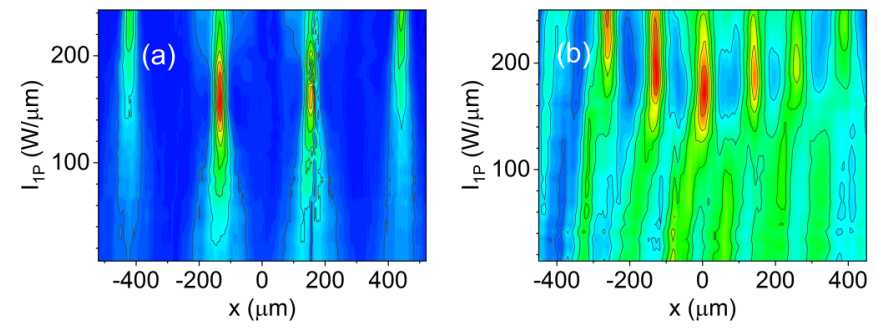

FIG. 4. Color-coded normalized intensity evolution of the growth-decay cycle of breathers with different spatial periods: (a) period $300 \mu \mathrm{m}, m=0.6, T=285^{\circ} \mathrm{C}$; (b) period $133 \mu \mathrm{m}, m=0.35$, $T=287.5^{\circ} \mathrm{C}$.

works well for the FF spatial dynamics in the cascading regimes with the applied large wave-vector mismatch and low SH fields, as proved by comparison to time-resolved calculations, even with a significant group velocity mismatch between the $\mathrm{FF}$ and the $\mathrm{SH}$ waves [35]. Figure 2 shows at low intensities linear propagation and at $\approx 50 \mathrm{~W} / \mu \mathrm{m}$ the initial stage of MI with exponentially amplified harmonic modulation. This growth dynamics has been characterized in previous studies $[8,26,28]$ and used for MI gain measurements. By increasing the intensity the amplification and compression saturate, the beams develop their maxima, and then a decay dynamics takes place as predicted by the Akhmediev breather solution. The decay state is disturbed by secondary maxima between the breather maxima [see also Fig. 3(d)]. This is an incipient higher-order breather $[36,37]$ because also higher harmonics of the breather pattern start to experience MI gain at intensities above $180 \mathrm{~W} / \mu \mathrm{m}$.

The comparison of measured output scans of the FF beam profile with numerical calculations for specific input intensities in Fig. 3 highlight the excellent quantitative agreement between experiments and simulations.

The color-coded intensity of a breather with a larger periodicity in Fig. 4(a) shows the stronger beam compression with the typical broad depletion areas between the maxima. The field zeros near the maxima are recognizable but faded by the time averaging. Very nicely the generation of the second sideband, missing in our one-sideband excitation, is visible in a breather with smaller period in Fig. 4(b). For intensities below the MI threshold of $100 \mathrm{~W} / \mu \mathrm{m}$ we see in principle the interference fringes between main and tilted input beams propagating with half the tilt angle. Therefore, the fringes at the input and output are shifted along $x$. With increasing intensity the shift decreases. When MI sets in above $100 \mathrm{~W} / \mu \mathrm{m}$ the position of the output maxima is locked close to the position of the input fringes indicating the formation of the second sideband.

Because the breathers develop on a 1.4-mm-wide Gaussian beam instead of an infinitely wide background, an intensity dependence of the position of different breather maxima in one row can be observed in Figs. 2 and 4. In the lower intensity edges of the beam higher input intensities are required to observe a breather maximum at the waveguide output [see also Fig. 5(b)].

We investigated Akhmediev breathers for the whole range of the interesting parameters: initial harmonic modulation
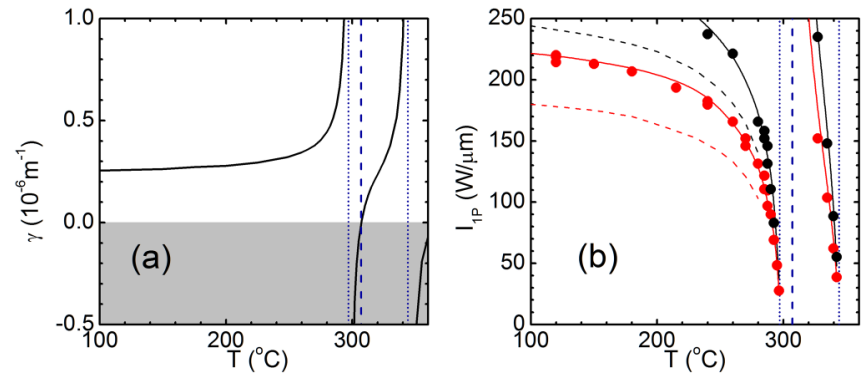

FIG. 5. (a) Effective cubic nonlinearity $\gamma$. (b) Input intensity $I_{1 \mathrm{P}}$ for the observation of the breather maxima at the waveguide output, modulation period $185 \mu \mathrm{m}, m=0.6$ for red objects, $m=$ 0.35 for dark objects. Circles refer to measurements, solid lines to simulations. The dashed lines show analytical predictions from [31]. Dotted blue vertical lines indicate phase-matching temperatures for $\mathrm{TE}_{0}$ and $\mathrm{TE}_{1}$ resonances. The dashed blue vertical line indicates the temperature where the different nonlinear contributions cancel each other.

strength and modulation period, and finally we changed the effectively acting cubic nonlinearity. By varying the wave-vector mismatch with temperature we control the nonlinear refraction parameter $\gamma$ with a dependence shown in Fig. 5(a). The decrease (increase) of the initial strength of the harmonic modulation increases (decreases) the spatial distance between the initial excitation and the breather maxima, thus the necessary input intensity for the observation of the breather maxima increases (decreases). This dependence is reported in Fig. 5(b) by comparing input modulation strengths of $m=0.6$ and $m=0.35$ at the fixed harmonic period of $185 \mu \mathrm{m}$. In particular, Fig. 5(b) shows the necessary input intensity for detecting the maxima of the breathers at the waveguide output. The measured intensity versus temperature $T$ - or nonlinearity $\gamma$-is compared to simulations and analytical predictions for $\mathrm{cw}$ beams. In our pulsed experiment the required intensity was $\approx 20 \%$ larger than the $\mathrm{cw}$ prediction from [31].

Breathers have been detected at all temperatures with a positive effective nonlinearity $\gamma$. As the SHG resonances are approached, the corresponding cascading effective nonlinearity increases and the input intensity for breather maxima at the output drastically decreases. Towards lower temperatures $<100^{\circ} \mathrm{C}$ the cascading contribution diminishes and the power for a breather maximum at the output would approach a value above $220 \mathrm{~W} / \mu \mathrm{m}$ of a pure $\chi^{(3)}$ breather. At the resonance temperatures $297^{\circ} \mathrm{C}$ and $344^{\circ} \mathrm{C}$, the SHG process dominates with strong $\mathrm{SH}$ conversion and breathers cannot be excited. At $307^{\circ} \mathrm{C}$, quadratic cascaded contributions of $\mathrm{TE}_{0}, \mathrm{TE}_{1}$, and the intrinsic cubic nonlinearity perfectly compensate and no nonlinear dynamics can be observed at all.

Conclusions. Our results represent the first deterministic experimental characterization of spatial Akhmediev breathers describing the growth-decay MI dynamics on a quasi-planewave background with an initial harmonic modulation in a quadratic waveguide. All details of the observed first row of highly localized light spots correspond qualitatively as well as quantitatively to the characteristics of the Akhmediev breather solution in the vicinity of its maxima, namely, the formation 
of a maximum between regions of MI growth and decay. Breathers have been demonstrated in a wide parameter range of positive net cascading nonlinearities and different initial harmonic modulation strengths and periods. The first row of light localization maxima was very robust against changing the system from very cubic-like for large wave-vector mismatch to the cascading regime, closer to phase matching. At phase matching with strong conversion to the $\mathrm{SH}$, no breatherlike beams were observed. Remarkably, these results lead to the reinterpretation of quadratic MI studies [8,25-28] in terms of shaping-invariant solitons and breathers, rather than only solitons, as main actors of complex MI phenomena. Moreover, the demonstration of Akhmediev breathers in the quadratic, not integrable system Eqs. (1) feeds the open discussion on FPU recurrence in Hamiltonian systems, as opposed to integrable systems only [34,38,39].

Acknowledgments. R.S. acknowledges the experimental support of F. Setzpfandt and T. Pertsch at the Institute of Applied Physics, Abbe Center of Photonics, Friedrich-SchillerUniversität Jena where the experiments have been performed. F.B. acknowledges C. De Angelis for fruitful theoretical discussion.
[1] E. Infeld and R. Rowlands, Nonlinear Waves, Solitons and Chaos (Cambridge University, Cambridge, England, 1990).

[2] V. I. Bespalov and V. J. Talanov, Pis'ma Zh. Eksp. Teor. Fiz. 3, 471 (1966) [JETP Lett. 3, 307 (1966)].

[3] T. B. Benjamin and J. E. Feir, J. Fluid Mech. 27, 417 (1967).

[4] V. E. Zakharov and L. A. Ostrovsky, Phys. D 238, 540 (2009).

[5] F. T. Arecchi and E. O. Schulz-DuBois, Laser Handbook (North-Holland, Amsterdam, 1972).

[6] K. Tai, A. Hasegawa, and A. Tomita, Phys. Rev. Lett. 56, 135 (1986).

[7] A. V. Mamaev, M. Saffman, D. Z. Anderson, and A. A. Zozulya, Phys. Rev. A 54, 870 (1996).

[8] R. A. Fuerst, D.-M. Baboiu, B. Lawrence, W. E. Torruellas, G. I. Stegeman, S. Trillo, and S. Wabnitz, Phys. Rev. Lett. 78, 2756 (1997).

[9] B. Kibler, J. Fatome, C. Finot, G. Millot, F. Dias, G. Genty, N. Akhmediev, and J. M. Dudley, Nat. Phys. 6, 790 (2010).

[10] B. Frisquet, B. Kibler, Ph. Morin, F. Baronio, M. Conforti, G. Millot, and S. Wabnitz, Sci. Rep. 6, 20785 (2016).

[11] M. Onorato, S. Residori, U. Bortolozzo, A. Montina, and F. T. Arecchi, Phys. Rep. 528, 47 (2013).

[12] J. M. Dudley, F. Dias, M. Erkintalo, and G. Genty, Nat. Photon. 8, 755 (2014).

[13] J. M. Soto-Crespo, N. Devine, and N. Akhmediev, Phys. Rev. Lett. 116, 103901 (2016).

[14] M. Guasoni, J. Garnier, B. Rumpf, D. Sugny, J. Fatome, F. Amrani, G. Millot, and A. Picozzi, Phys. Rev. X 7, 011025 (2017).

[15] C. Bao, J. A. Jaramillo-Villegas, Y. Xuan, D. E. Leaird, M. Qi, and A. M. Weiner, Phys. Rev. Lett. 117, 163901 (2016).

[16] N. Akhmediev and V. I. Korneev, Theor. Math. Phys. 69, 1089 (1986).

[17] B. Kibler, A. Chabchoub, A. N. Gelash, N. Akhmediev, and V. E. Zakharov, Phys. Rev. X 5, 041026 (2015).

[18] M. Närhi, B. Wetzel, C. Billet, S. Toenger, T. Sylvestre, J. M. Merolla, R. Morandotti, F. Dias, G. Genty, and J. M. Dudley, Nat. Commun. 7, 13675 (2016).

[19] A. Mussot, C. Naveau, M. Conforti, A. Kudlinski, F. Copie, P. Szriftgiser, and S. Trillo, Nat. Photon. 12, 303 (2018).

[20] C. Naveau, P. Szriftgiser, A. Kudlinski, M. Conforti, S. Trillo, and A. Mussot, Opt. Lett. 44, 763 (2019).
[21] D. Pierangeli, M. Flammini, L. Zhang, G. Marcucci, A. J. Agranat, P. G. Grinevich, P. M. Santini, C. Conti, and E. DelRe, Phys. Rev. X 8, 041017 (2018).

[22] R. Schiek, J. Opt. Soc. Am. B 10, 1848 (1993).

[23] F. Baronio, Opt. Lett. 42, 1756 (2017).

[24] F. Baronio, S. Chen, and D. Mihalache, Opt. Lett. 42, 3514 (2017).

[25] S. Trillo and P. Ferro, Opt. Lett. 20, 438 (1997).

[26] H. Fang, R. Malendevich, R. Schiek, and G. I. Stegeman, Opt. Lett. 25, 1786 (2000).

[27] R. Schiek, Y. Baek, and G. I. Stegeman, Phys. Rev. E 53, 1138 (1996).

[28] R. Schiek, H. Fang, R. Malendevich, and G. I. Stegeman, Phys. Rev. Lett. 86, 4528 (2001).

[29] $d=d_{31}=\chi_{z y y}^{(2)}=-3.63 \times 10^{-23} \frac{\mathrm{As}}{\mathrm{V}^{2}}, \quad \chi^{(3)}=\chi_{y y y y}^{(3)}=3.2 \times$ $10^{-32} \frac{\mathrm{Asm}}{\mathrm{V}^{3}} ; K^{(2)}=1.8 \times 10^{6} \frac{\mathrm{V}^{3}}{\mathrm{~m}^{2}}$ for the $\mathrm{TE}_{0}$ resonance; $K^{(2)}=$ $2.0 \times 10^{6} \frac{\mathrm{V}^{3}}{\mathrm{~m}^{2}}$ for the $\mathrm{TE}_{1}$ resonance; $K^{(3)}=1.9 \times 10^{10} \frac{\mathrm{V}^{4}}{\mathrm{~m}^{3}}$; $\alpha_{1}=0.04 \mathrm{~cm}^{-1}, \alpha_{2}=0.08 \mathrm{~cm}^{-1}$.

[30] R. Schiek, Y. Baek, and G. I. Stegeman, J. Opt. Soc. Am. B 15, 2255 (1998).

[31] M. Erkintalo, G. Genty, B. Wetzel, and J. M. Dudley, Phys. Lett. A 375, 2029 (2011).

[32] J. M. Soto-Crespo, N. Devine, and N. Akhmediev, Phys. Rev. A 96, 023825 (2017).

[33] S. Trillo (private communication).

[34] P. G. Grinevich and P. M. Santini, Phys. Lett. A 382, 973 (2018).

[35] P. H. Pioger, V. Couderc, L. Lefort, A. Barthelemy, F. Baronio, C. De Angelis, Y. Min, V. Quiring, and W. Sohler, Opt. Lett. 27, 2182 (2002).

[36] S. Wabnitz and N. Akhmediev, Opt. Commun. 283, 1152 (2010).

[37] M. Erkintalo, K. Hammani, B. Kibler, C. Finot, N. Akhmediev, J. M. Dudley, and G. Genty, Phys. Rev. Lett. 107, 253901 (2011).

[38] O. Kimmoun, H. C. Hsu, H. Branger, M. S. Li, Y. Y. Chen, C. Kharif, M. Onorato, E. J. R. Kelleher, B. Kibler, N. Akhmediev, and A. Chabchoub, Sci. Rep. 6, 28516 (2016).

[39] A. E. Kraych, P. Suret, G. El, and S. Randoux, Phys. Rev. Lett. 122, 054101 (2019). 